\title{
An Novel Atomic Scalar Magnetometer Using Laser
}

\author{
Chao Wang, Zhijian Zhou, and Defu Cheng
}

\begin{abstract}
The measurement precision of commercial atom scalar magnetometer is relatively backward compared with that of quantum magnetometer. However, the application of quantum magnetometers such as SERF requires more stringent environmental background requirements, which is not suitable for magnetic field measurement in the geomagnetic environment. The purpose of this paper is to design a ${ }^{4} \mathrm{He}$ atom scalar magnetometer using ECDL laser. Compared with the conventional atomic scalar magnetometer, this magnetometer has higher measuring precision and can work normally in the geomagnetic environment. In order to achieve the above goals, the sensitivity formula of the atomic scalar magnetometer is first deduced and calculated, and the key physical factors that directly affect the sensitivity are the optical pumping rate, transverse relaxation rate, and longitudinal relaxation rate. Then, the light source and ${ }^{4} \mathrm{He}$ cell are determined as key components which affect sensitivity. On this basis, the optical path of the ${ }^{4} \mathrm{He}$ atomic scalar magnetometer using laser is designed in this paper. The light path ensures the stability of the laser wavelength of $1083.207 \mathrm{~nm}$ by the saturation absorption spectrum method, and it ensures the circularly polarized light enters the ${ }^{4} \mathrm{He}$ cell through the combination of various optical components. This paper also studies the electric excitation technology of the ${ }^{4} \mathrm{He}$ cell. And, combined with simulation experiments, the High-Frequency discharge excitation circuit with high energy transfer efficiency and corresponding matching network are determined. Through the optical wavelength meter, it can be determined that the optical path designed in this paper can guarantee the wavelength stability of $1083.207 \mathrm{~nm}$ for a long time. By analyzing the detection signals of PD, the circularly polarized light enters the ${ }^{4} \mathrm{He}$ cell in the light circuit designed in this paper has a higher degree of polarization. The High-Frequency discharge excitation circuit designed in this paper can light up the cell smoothly, and the input power when the circuit works stably is about $6 \mathrm{~W}$. Finally, the static sensitivity of the magnetometer is $5 \mathrm{pT} / \mathrm{Hz}^{1 / 2}$. The ${ }^{4} \mathrm{He}$ atom scalar magnetometer using ECDL laser designed in this paper has high static sensitivity, which basically meets the design requirements, and the instrument can be used normally in the geomagnetic environment. However, the instrument still has a lot of room for improvement, including optical path and cell performance optimization, and we will continue to study in this direction.
\end{abstract}

Keywords — Scalar magnetometer, laser, resonance signal, excitation circuit.

This work was supported by the Special Projects for Monitoring, Warning and Prevention of Major Natural Disasters, the National Key Research and Development Program of China 2018 (Grant No. 2018YFC1503803 and 2018YFC1503903).

C. Wang is with the College of Instrumentation and Electrical Engineering, Jilin University, Changchun, China (e-mail: chaow15@mails.jlu.edu.cn)

Z. Zhou is with the College of Instrumentation and Electrical Engineering, Jilin University, Changchun, China (e-mail: zhouzhijian@jlu.edu.cn)

D. Cheng is with the College of Instrumentation and Electrical Engineering, Jilin University, Changchun, China (e-mail: chengdefu@jlu.edu.cn)

\section{INTRODUCTION}

S INCE the nuclear magnetic resonance (NMR) findings in $\checkmark 1946$ and the first atomic magnetometer developed in 1961

[1], the research of atomic magnetometers has made rapid progress in recent years [2]. This class of sensors has extensively applied in fields including space exploration, geophysical exploration and military anti-submarine [3]. In the last two or three decades, we found him able to play an important role in predict earthquakes, even in biomagnetism and latent disease diagnosis [4].

In the field of disease diagnosis, Magnetocardiogram (MCG) and magnetoencephalogram (MEG) can be generated by detecting magnetic information in human tissues such as the heart and brain, in turn, the latent lesion of human tissue is detected in time. The strength of the magnetic field in the body's main organs is shown in Table.I [5]. Magnetoencephalography (MEG) was studied with optically pumped magnetometer by the researchers of Okayama University [6].

Spin Exchange Relaxation Free (SERF) magnetometers have a sensitivity of $1-10 \mathrm{fT} / \mathrm{Hz}^{1 / 2}$, it is the most sensitive atomic scalar magnetometers at presented, nevertheless, the work environment in near-zero magnetic field it needs is particularly harsh [7]. It is not suitable for the measurement of the earth's magnetic field [8].

This paper introduces a kind of optically pumped method ensures the stability of laser wavelength. With reasonable optical path construction and efficient design of HF discharge excitation circuit, we have a magnetometer reaches a sensitivity of $5 \mathrm{pT} / \mathrm{Hz}^{1 / 2}$ in a static measurement.

TABLE I. The strength of the magnetic field in the body's main organs.

\begin{tabular}{c|c}
\hline \hline Organ name & Magnetic field intensity(pT) \\
\hline \hline heart (MCG) & 50 \\
\hline brain (MEG) & 1 \\
\hline muscle (MMG) & 10 \\
\hline lung (MPG) & 5000 \\
\hline eye(MOG) & 10 \\
\hline retina(MPG) & 0.1 \\
\hline \hline
\end{tabular}

\section{II. ${ }^{4} \mathrm{HE}$ ATOMIC MAGNETOMETER SENSITIVITY}

Briefly, the ${ }^{4} \mathrm{He}$ atomic magnetometer we introduced, is a magnetic measuring instrument on the basis of Zeeman effect in the magnetic field, optical pumping, and NMR. 
Usually, we study sensitivity by the quality of the resonance signal from the photodetector shown in Fig.1(a), the sensitivity of ${ }^{4} \mathrm{He}$ optically pump magnetometer can be expressed by [9]

$$
\delta \mathrm{B}=\frac{1 \Delta \mathrm{f}}{\gamma \mathrm{S}_{\mathrm{e}} \mathrm{N} /}
$$

$\delta \mathrm{B}$------ Sensitivity of magnetometer.

$\gamma_{\mathrm{He}}$------ Helium atomic rotation ratio.

$\Delta \mathrm{f}$------ Line width of resonance signal.

$\mathrm{S}$------ Amplitude of resonance signal.

$\mathrm{N}$------ Noise level of resonance signal.
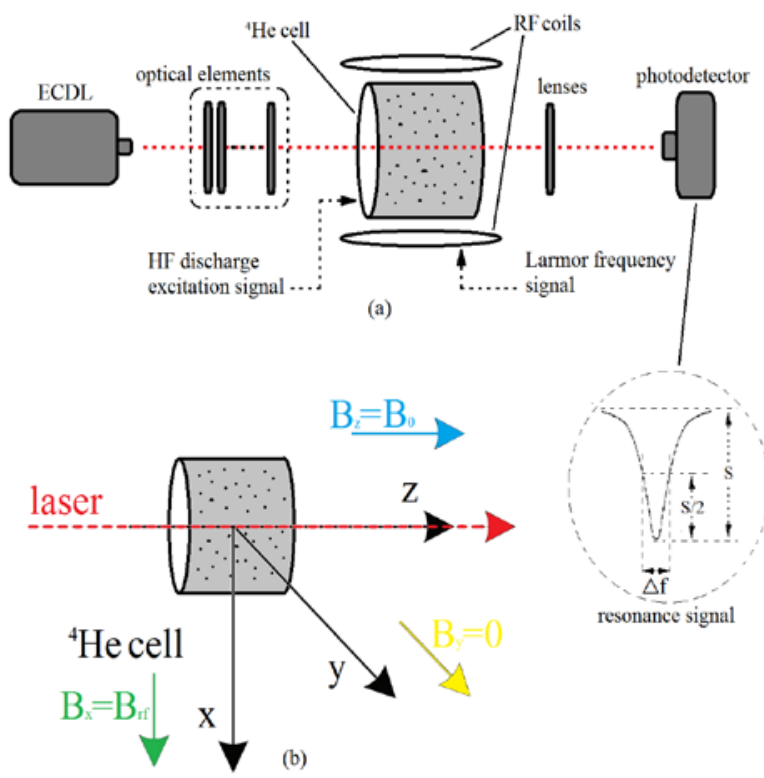

Fig. 1. Experimental coordinates system and laser, magnetic field, rf field direction

With the magnetic field and light conditions shown in Fig.1(b), we can construct an optical Bloch equation that considers the combined effects of optical pumping and relaxation. It is expressed by

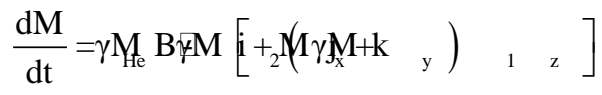

$$
\begin{aligned}
& +\left[-\left[-M\left(i-M_{x} \Gamma j M_{y}-\right) M_{p} k\left(\begin{array}{lll} 
& & \\
&
\end{array}\right)\right]\right.
\end{aligned}
$$

$\gamma_{2}$------ Transverse relaxation rate.

$\gamma_{1}$------ Longitudinal relaxation rate.

$\Gamma_{\mathrm{P}}$------ Optical pumping rate.

$M=\left(M_{x}, M_{y}, M_{z}\right)$------ The total magnetic moment of the

${ }^{4} \mathrm{He}$ and its components in three directions.

$\mathrm{B}=\left(\mathrm{B}_{\mathrm{x}}, \mathrm{B}_{\mathrm{y}}, \mathrm{B}_{\mathrm{z}}\right) \oplus \mathrm{\oplus}\left(\mathrm{DBB} \operatorname{BCos} \quad{ }_{0}\right)$------ The total magnetic

field value and its components in three directions.

Equation (2) can be obtained as (3) with the basic algorithm of determinants.

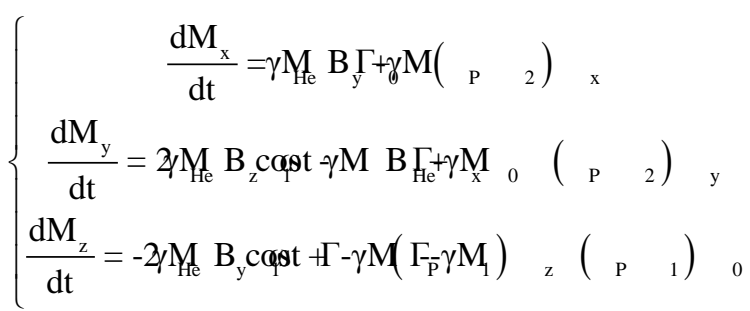

On the basis of the coordinate system $\mathrm{S}(\mathrm{x}, \mathrm{y}, \mathrm{z})$ shown in Fig.1(b), the rotating coordinate system $\mathrm{S}^{\prime}$ ( $\left.\mathrm{x}^{\prime}, \mathrm{y}^{\prime}, \mathrm{z}^{\prime}\right)$ is introduced to revolve around the z-axis with frequency $\omega$. The Larmor precession frequency goes from $\omega 0$ to $(\omega 0-\omega)$. In the rotating coordinate system, (3) can be expressed by

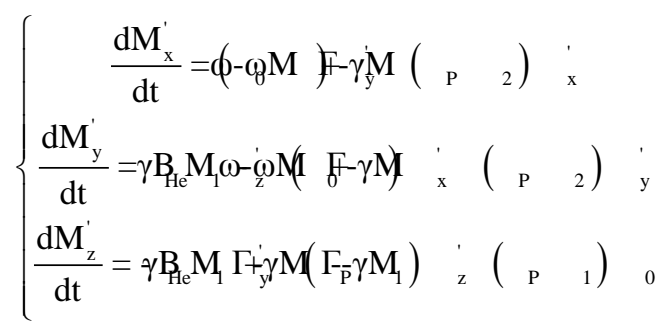

With all conditions, the macroscopic magnetic moment will eventually reach the equilibrium state. So

$$
\frac{\mathrm{dM}_{\mathrm{x}}^{\prime}}{\mathrm{dt}}=\frac{\mathrm{dM}_{\mathrm{y}}^{\prime}}{\mathrm{dt}}=\frac{\mathrm{dM}_{\mathrm{z}}^{\prime}}{\mathrm{dt}}=0
$$

When the laser direction is combined with the $\mathrm{z}\left(\mathrm{z}^{\prime}\right)$ axis, the optical power of the photodetector is represented by magnetic moment on the $z\left(z^{\prime}\right)$ axis, expressed by

$$
\mathrm{M}_{\mathrm{z}}^{\prime}=\frac{\left[\frac{\left(\omega_{\overline{0}} \omega\right)^{2}}{\Gamma_{\overline{\mathrm{p}}} \gamma}+\left(-\gamma_{\mathrm{p}} \mathrm{M}_{2}\right)\right]}{0}
$$

And

$$
\omega_{0}=\gamma \mathrm{B}_{\text {Не }} \quad 0
$$

The amplitude of the resonant signal in (1) is defined as the difference between Max and min of magnetic moment on the $z\left(z^{\prime}\right)$ axis, shown in Fig.1(a). And, the line width of resonance signal in (1) is defined as the difference between two $\omega$ values corresponding to the half signal peak, shown in Fig.1(a). The result is calculated and simplified, can be expressed by

$$
\begin{gathered}
\Delta \mathrm{f} \approx \frac{\gamma \mathrm{B}_{\mathrm{Re}}}{\pi} \sqrt{\frac{\left(\Gamma_{\overline{\mathrm{p}}} \gamma_{2}\right)}{\left(\Gamma_{\overline{\mathrm{p}}} \gamma_{1}\right)}} \\
\mathrm{S} \approx \frac{\Gamma_{\mathrm{P}}}{\Gamma_{\overline{\mathrm{p}} \gamma}} \mathrm{M}_{0}
\end{gathered}
$$

Optical pumping rate, transverse relaxation rate, and longitudinal relaxation rate are physical quantities directly 


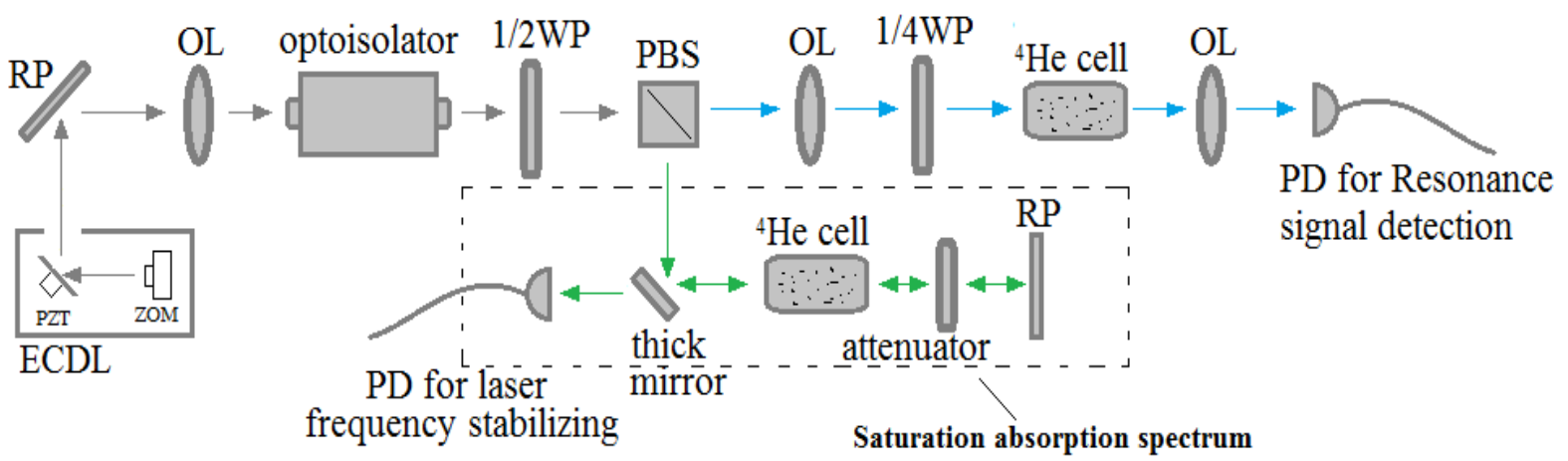

Fig. 2. ${ }^{4} \mathrm{He}$ atomic scalar magnetometer optical system structure

related to the physical properties of the light source and ${ }^{4} \mathrm{He}$ cell. (8) and (9) indicates that the quality of the light source and ${ }^{4} \mathrm{He}$ cell determine the sensitivity of the magnetometer.

\section{III. ${ }^{4}$ HE ATOMIC SCALAR MAGNETOMETER OPTICAL DESIGN}

The measurement standard of light quality mainly includes two points, namely the intensity and the stability of light. For the measurement of ${ }^{4} \mathrm{He}$ atom scalar magnetometer we studied, the effective wavelength of the light source is around $1083.207 \mathrm{~nm}$. So, we need to make sure that we can get a $1083.207 \mathrm{~nm}$ single-frequency laser with a strong and stable wavelength.

The exciting radiation of the luminescent medium particles in the laser formed the laser beam [10]. A single frequency (wavelength) laser must be polarized because the stimulated radiation has a distinctive feature: the photon produced by radiation has the same phase, the same propagation direction, and the same polarization state as the external photon [11]. Moreover, the ECDL used by us has a resonant cavity. There are two Brewster windows at both ends of the cavity. The laser will reflect back and forth in the resonator. Assuming a three-dimensional axis, and the direction of laser propagation is in the z-direction. The laser will reflect some of the linearly polarized light along the $\mathrm{x}$-direction every time meet Brewster window (The design of Brewster Window makes each incident angle a Brewster Angle [12]). After multiple reflections, the linearly polarized light in the $\mathrm{x}$-direction will be completely reflected off. The final transmission will be the pure line polarized light in the y-direction [13].

In order to improve the performance of ${ }^{4} \mathrm{He}$ atomic scalar magnetometer, the pump light used should be circularly polarized light [14]. We need to design an optical path with both optical frequency stabilization and polarization adjustment function.

We designed the optical system as shown in Fig.2. Do the following definition in the Fig.2. ECDL is external cavity diode laser. RP is reflector plate. $1 / 2 \mathrm{WP}$ and $1 / 4 \mathrm{WP}$ are $1 / 2$ wave plate and $1 / 4$ wave plate respectively. PBS is a polarized beam splitter. OL is the optical lens. PD is a photodetector.

After shaping through OL, the parallel laser beam passes through the optoisolator, and then it is divided into transmission beam and reflected beam through the combination of $1 / 2 \mathrm{WP}$ and PBS. Reflection beam is used for saturation absorption spectrum. Because RP and attenuator, the intensity of light in the two directions of ${ }^{4} \mathrm{He}$ cell will be very different so that PD can get the absorption peak signal needed to achieve the laser frequency stabilization function. Transmission beam is used for the measurement of the magnetometer. OL further ensures the collimation of the beam, and previous optical elements did not change the polarization state of the beam, transmission beam is still linear polarized light. With 1/4 WP, transmission beam can be converted to circularly polarized light.

In order to examine the polarization of transmitted beam, a test system was designed using an analyzer and PD based on the beam path of Fig.2 shown in Fig.3. The analyzer can rotate around the direction of the beam. Observe the output signal of Observe PD output signal, the transmitted beam is a high quality circularly polarized light when the analyzer is rotated $360^{\circ}$ and the signal strength remains unchanged [15].

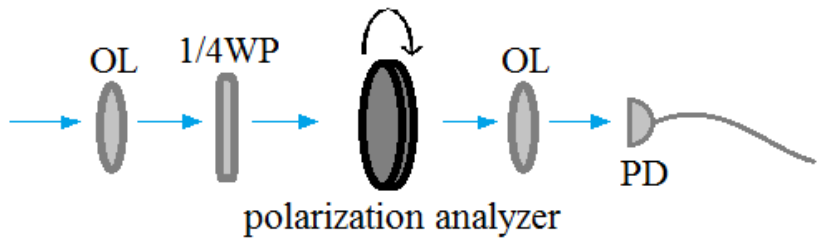

Fig. 3. Polarization test system structure

We used the optical wavelength meter to monitor the output laser of the optical path several times for 1 hour, and the results showed that the saturated absorption spectrum method can guarantee the laser with a stable wavelength of $1083.207 \mathrm{~nm}$ for a long time. And, through the analysis of the PD signals, we find that in the process of polarization analyzer $360^{\circ}$ rotation, signal strength almost able to keep a straight line, that can produce light path magnetometer need circularly polarized light.

The ${ }^{4} \mathrm{He}$ atomic scalar magnetometer is based on optical pump and NMR. These two physical effects cannot occur in the ground state of ${ }^{4} \mathrm{He}$. But the atoms in the metastable state can satisfy the basic conditions required for the above physical effects. We need an HF excitation circuit to ignited inside a cell filled with ${ }^{4} \mathrm{He}$. This discharge ensures ${ }^{4} \mathrm{He}$ at ground state can be excited to a metastable state, shown as Fig.4. 


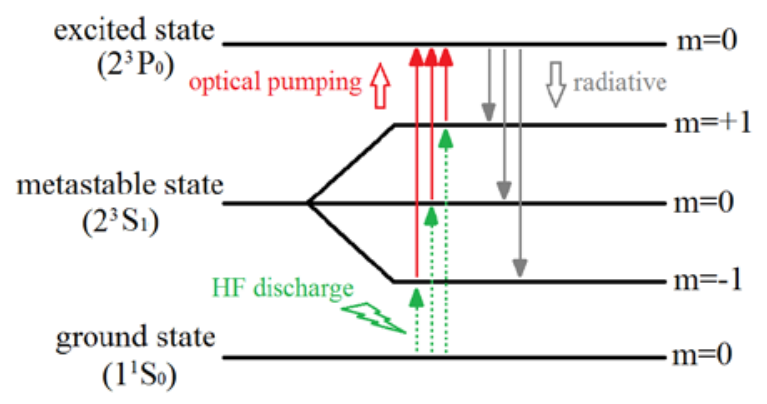

Fig. 4. Polarization test system structure

The dynamic equilibrium of optical pumping and radiation can be arranged in an atomic orientation, then, NMR occurs when the frequency of the $B_{\text {rf }}$ shown in Fig.1 and the Larmor frequency of the magnetic moment precession match each other. We can calculate the value of the measured magnetic field by the frequency of $\mathrm{B}_{\mathrm{rf}}$ and $\gamma_{\mathrm{He}}$.

The cell filled with ${ }^{4} \mathrm{He}$ is a key component of magnetic measurement by the ${ }^{4} \mathrm{He}$ atomic scalar magnetometer. The cell can be seen as a cylindrical gas discharge lamp that can be illuminated by the external electrodes. Through the Francis equation [16], shown as (10) and (11), it can be shown that the cell exhibits stable resistance properties in the steady state after the internal gas is ignited. The parameters in the (10) and (11) include cell voltage $\mathrm{v}$, cell current $\mathrm{i}$, average electron concentration N, and cell resistance R. A, B and F are constants. It is necessary to design a circuit that can generate an $\mathrm{HF}$ excitation signal and transmit the energy of the signal efficiently to the cell.

$$
\begin{gathered}
\frac{\mathrm{dN}}{\mathrm{dt}}=\mathrm{A} \cdot \mathrm{i} \cdot \mathrm{v}-\mathrm{B} \cdot \mathrm{N} \\
\mathrm{R}=\frac{\mathrm{v}}{\mathrm{i}}=\frac{\mathrm{F}}{\mathrm{N}}
\end{gathered}
$$

We designed an HF excitation circuit for the ${ }^{4} \mathrm{He}$ cell shown in Fig.5. An active crystal oscillator was used to produce a $50 \mathrm{MHz}$ HF signal, then a power amplifier is designed with the Metal Oxide Semiconductor (MOS). The DC bias and the signal amplitude of the signal input into power amplifier can be adjusted by changing the value of the R3 (shown in Fig.5) to ensure the circuit work in the best state.

The cell is installed inside the magnetometer probe, which is far from the signal generating circuit. The excitation signal needs to be transmitted to cell through a $50 \Omega$ coaxial cable, so we designed a T-network between the power amplifier and coaxial cable with an inductance (L3, shown in Fig.5) and capacitance (C9, C10, C11, C12, shown in Fig.5) to match the impedance.

The output impedance of the amplifier is $10 \Omega$. The impedance of the coaxial transmission line is $50 \Omega$. In the ideal case, calculation by the relevant formula of T-network, we get that $\mathrm{L} 3=636.62 \mathrm{nH}, \mathrm{C} 9\|\mathrm{C} 10=7.15 \mathrm{pF}, \mathrm{C} 11\| \mathrm{C} 12=15.9 \mathrm{pF}$.

Simulated by Advanced Design System (ADS), results are shown in Fig.6. At $50 \mathrm{MHz}, \mathrm{dB}\left(\mathrm{S}_{11}\right)=-66.232 \mathrm{~dB}$ ( $\mathrm{m} 1$ in Fig.6), $\mathrm{dB}\left(\mathrm{S}_{21}\right)=0 \mathrm{~dB}$ ( $\mathrm{m} 2$ in Fig.6), $3 \mathrm{~dB}$ bandwidth $\mathrm{BW}_{0.7} \approx 4.4 \mathrm{MHz}$. Fig.6 proved this design has achieved the best match.

There is a receiving circuit of HF signal near cell as shown in Fig.6. The value of the cell is about $500 \Omega$ higher than the $50 \Omega$ of coaxial, the input impedance needs to be raised with a self-coupled coil, and an LC parallel resonant network composed of the self-coupled coil and the capacitance is designed to do $50 \mathrm{MHz}$ frequency selection.

When the ${ }^{4} \mathrm{He}$ cell was ignited, the total input power is about $6 \mathrm{~W}$.

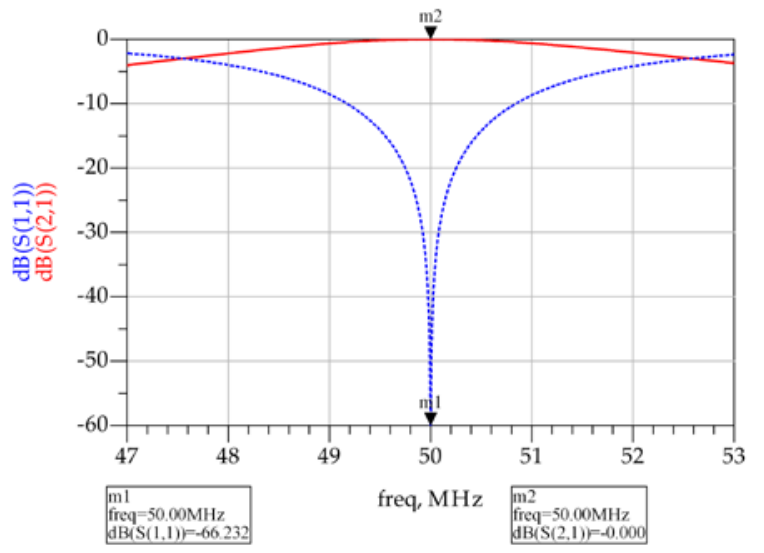

Fig. 6. Simulation results of T-network in circuit

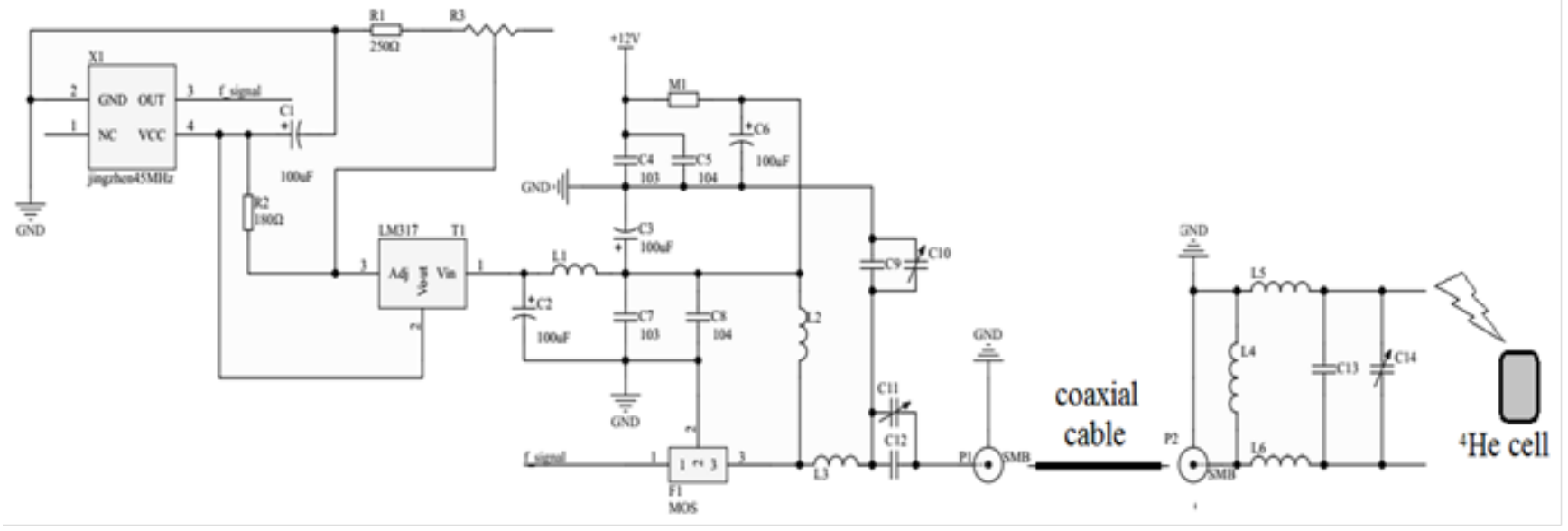

Fig. 5. The HF excitation circuit for the ${ }^{4} \mathrm{He}$ cell 


\section{PERFORMANCE OF ${ }^{4}$ HE ATOMIC SCALAR MAGNETOMETER}

Use the magnetic shielding tube shown in Fig.7(a) to create a theoretical "zero magnetic" space. The tube is a 6-layer structure, one end is sealed, the other end is a flexible cover, the inner layer is an epoxy tube, the outer layer is an aluminum layer, and the middle layer is a 4-layer magnetic conductive permalloy alloy. The attenuation of external geomagnetic field by the tube can reach about $80 \mathrm{~dB}$.

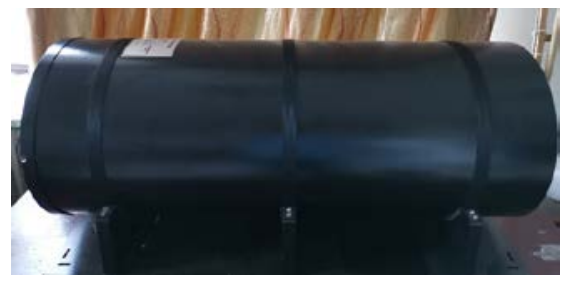

(a)

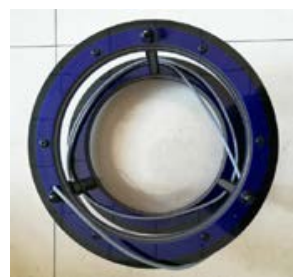

(b)
Fig.7. (a) Magnetic shielding tube (b) Helmholtz coils.

Helmholtz coil, as shown in Fig.7(b), are used to create different types of stable magnetic field environments in the magnetic shielding tube. The strength and direction of the magnetic field can also be controlled by the current passing through the coils. The Helmholtz coils with a $15 \mathrm{~cm}$ radius and 217 turns. According to the simulation results, the proportional coefficient of magnetic field to current in the uniform region of Helmholtz coils is about $540 \mathrm{nT} / \mathrm{mA}$.

According to the collected data, the resonance signal curve is drawn as shown in Fig.8. The solid red line represents the signal measured by the magnetometer of the light source of the gas discharge lamp, and the solid blue line represents the signal measured by the magnetometer of the laser light source. Only light source replacement was done in the two tests, other conditions were identical.

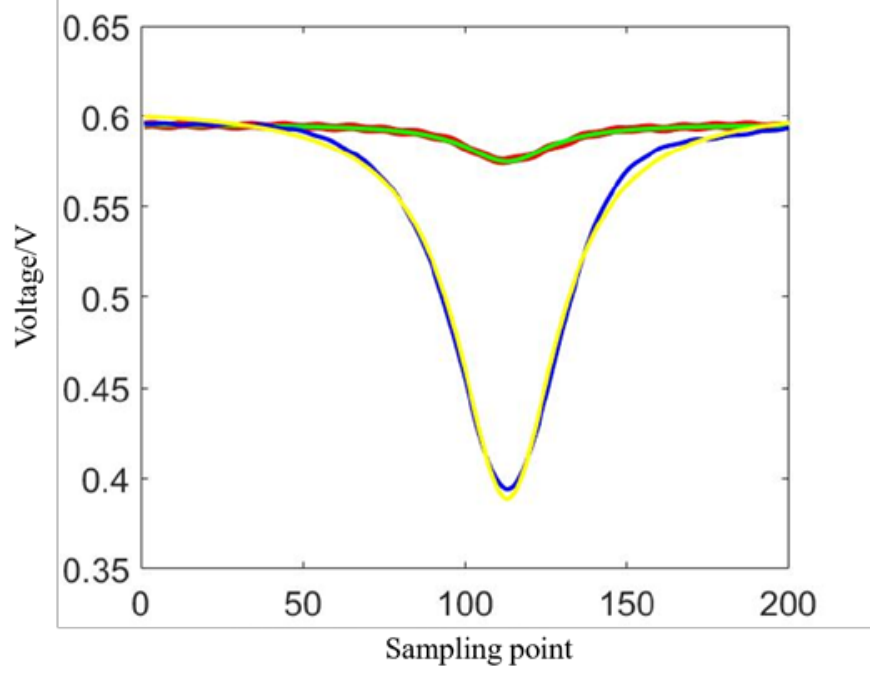

Fig. 8. The resonance signal contrast of laser magnetometer and gas lamp magnetometer

According to the calculation of scan frequency range, scan period and data sampling rate, the corresponding scanning frequency of each sampling interval is about 1000Hz.Fig.8 ordinate is the sampling voltage value in unit $\mathrm{V}$.
Lorentz line fitting was performed on the test data and the curve was also shown in Fig.8 after the horizontal coordinate was shifted. The solid green line represents the fitting curve of the magnetic sensor data of the light source of the gas discharge lamp, and the solid yellow line represents the fitting curve of the magnetic sensor data of the laser light source. Two values of signal line width $\Delta \mathrm{f}$ and absorption peak amplitude $\mathrm{S}$ are further calculated as shown in Table II.

It is concluded that the sensitivity of the magnetic sensor can be increased by about 10 times after using the laser instead of the gas discharge lamp as the light source of the magnetometer.

We also measured the noise level value of the resonance signal is about $1.44 \mu \mathrm{V} / \mathrm{Hz}^{1 / 2} @ 1 \mathrm{~Hz}$, with (1), the laser ${ }^{4} \mathrm{He}$ atomic scalar magnetometer attained a sensitivity of about $5 \mathrm{pT} / \mathrm{Hz}^{1 / 2}$ in static measurement.

TABLE II. Two values of $\Delta \mathrm{f}$ and $\mathrm{S}$.

\begin{tabular}{c|c|c|c}
\hline \hline & $\Delta \mathrm{f}$ & $\mathrm{S}$ & $\Delta \mathrm{f} / \mathrm{S}$ \\
\hline \hline Magnetometer with lamp & $660 \mathrm{nT}$ & $20 \mathrm{mV}$ & 33.00 \\
\hline Magnetometer with laser & $768 \mathrm{nT}$ & $217 \mathrm{mV}$ & 3.54 \\
\hline \hline
\end{tabular}

\section{CONCLUSION AND PERSPECTIVES}

The quality of the light source and ${ }^{4} \mathrm{He}$ cell determine the sensitivity of the ${ }^{4} \mathrm{He}$ atomic scalar magnetometer. We ensure that these two factors can meet the requirements of the magnetometer through the reasonable design of the optical path and the circuit, respectively. In general, the magnetometer performs well.

${ }^{4} \mathrm{He}$ atomic scalar magnetometer is relatively mature in practical application, there will be much room for development. We will continue our efforts to make in-depth studies on the circular polarization of laser beam, the size of the cell, and the measurement dead zone.

\section{ACKNOWLEDGMENT}

This work was supported by the Special Projects for Monitoring, Warning and Prevention of Major Natural Disasters, the National Key Research and Development Program of China 2018 (Grant No. 2018YFC1503803 and 2018YFC1503903). The authors would like to thank the Key Laboratory of Geophysical Exploration Equipment ( Jilin University ) , Ministry of Education for their additional assistance.

\section{REFERENCES}

[1] A. R. Keyser, J. A. Rice, L. D. Schearer, “A metastable helium magnetometer for observing small geomagnetic fluctuations”, Journal of Geophysical Research, vol. 66, no.12, pp. 4163-4169, 1961.

[2] H. B. Dong, C. D. Zhang, “A Further Review of the Quantum Magnetometers”, Chinese Journal of Engineering Geophysics, vol. 7, no.4, pp. 460-470, 2010.

[3] A. Weis, et al. "Optically pumped alkali magnetometers for biomedical applications”. Europhysics News. vol. 43, no.3, pp. 20-23, 2012. 
[4] Y. Ito, H. Ohnishi, K. Kamada, et al. "Development of an optically pumped atomic magnetometer using a K-Rb hybrid cell and its application to magnetocardiography”. Aip Advances, vol. 2, no.3, pp. 325, 2012.

[5] T. Uchiyama, S. Nakayama, K. Mohri, et al. "Biomagnetic field detection using very high sensitive MI sensor for medical application”. Physical Status Solidi Applications \& Materials, vol. 206, no.4, pp.639-643. 2009.

[6] Shuji. Taue, Yasuyuki. Sugihara, Tetsuo. Kobayashi. "Development of a Highly Sensitive Optically Pumped Atomic Magnetometer for Biomagnetic Field Measurements: A Phantom Study”. IEEE TRANSACTIONS ON MAGNETICS, vol. 46, no.9, pp. 3635-3638, 2010.

[7] Z. Epstein, P. Sprangle, "An Optical Magnetometry Mechanism Above the Surface of Seawater”. IEEE Journal of Quantum Electronics, vol. 52, no.6, pp. 1-6, 2016.

[8] Y. Ito, H. Ohnishi, K. Kamada, et al. "Sensitivity Improvement of Spin-Exchange Relaxation Free Atomic Magnetometers by Hybrid Optical Pumping of Potassium and Rubidium”. IEEE Transactions on Magnetics, vol. 47, no.10, pp. 3550-3553, 2011.

[9] Y. Gu, R. Y. Shi, Y. H. Wang. "Study on sensitivity-related parameters of distributed feedback laser-pumped cesium atomic magnetometer”. Acta Physica Sinica, vol. 63, no.11, pp. 110701-110760, 2014.

[10] Yu. T, K. Hirose, T. Sugiyama, et al. "Principle of beam generation in on-chip 2D beam pattern projecting lasers”. Optics Express, vol. 26, no.8, pp. 10787, 2018.

[11] A. Yousef-Zamanian, M. Neshat. "Investigation of polarization state of terahertz radiation from compact laser-induced plasma in air". Optica Acta International Journal of Optics, vol. 64, no.3, pp. 300-308, 2017.

[12] O. Abida, J. Du, L. Zhu. Yousef-Zamanian, M. Neshat. "Investigation of the photolysis of the surface-adsorbed HNO 3, by combining laser photolysis with Brewster angle cavity ring-down spectroscopy". Chemical Physics Letters, vol. 534, no.5, pp. 77-82, 2012.

[13] T. Mazza, M. Ilchen, A. J. Rafipoo, et al. "Determining the polarization state of an extreme ultraviolet free-electron laser beam using atomic circular dichroism”. Nature Communications, vol. 5, no.4, pp. 3648, 2014.

[14] J. Rutkowski, W. Fourcault, F. Bertrand, et al. "Towards a Miniature Atomic Scalar Magnetometer Using a Liquid Crystal Polarization Rotator”. Sensors \& Actuators A Physical, vol. 216, no.3, pp. 386-393, 2014.

[15] D. C. Abeysinghe, Q. Zhan, R. L. Nelson, et al. "Spiral Plasmonic Lens as Miniature Circular Polarization Analyzer”. Optics Letters, vol. 34, no.20, pp. 3047-349, 2010.

[16] A. V. Pipa, J. Koskulics, R. Brandenburg, et al, "The simplest equivalent circuit of a pulsed dielectric barrier discharge and the determination of the gas gap charge transfer”, Review of Scientific Instruments, vol. 83, no.11, pp. 115112-115112-7, 2012. 